\title{
FREE PRODUCTS OF HYPERFINITE VON NEUMANN ALGEBRAS AND FREE DIMENSION
}

\author{
Ken DYKema \\ University of California, \\ Berkeley, California, USA 94720, \\ (e-mail dykema@math.berkeley.edu)
}

April 1992

\section{Introduction.}

Voiculescu's theory of freeness in noncommutative probability spaces (see $[10,11,12,13,14,15]$, especially the latter for an overview) has made possible the recent surge of results about and related to the free group factors $L\left(\mathbf{F}_{n}\right)[13,3,7,8,9,4]$. One hopes to eventually be able to solve the old isomorphism question, first raised by R.V. Kadison in the 1960's, of whether $L\left(\mathbf{F}_{n}\right) \cong L\left(\mathbf{F}_{m}\right)$ for $n \neq m$.

In Voiculescu's theory, (see also [1]), one takes free products of finite von Neumann algebras, denoted $\mathcal{A} * \mathcal{B}$, and one has $L(G) * L(H) \cong L(G * H)$, (where $L(G)$ for $G$ a discrete group is the group von Neumann algebra, which is generated by the left regular representation of $G$ on $\left.l^{2}(G)\right)$. It is of intrinsic interest to decide when $\mathcal{A} * \mathcal{B}$ is a factor, and to determine its isomorphism class. It may be that such results or the techniques used will give insight into the isomorphism problem. Moreover, such free products are related to amalgamated free products, which have arisen in connection with results about irreducible subfactors $\left[6,9,1^{\prime}\right]$.

In [4] and [9], the interpolated free group factors $L\left(\mathbf{F}_{r}\right)(1<r \leq \infty)$ were found, that have equality with the free group factor on $n$ generators if $r=n \in \mathbf{N} \backslash\{0,1\}$ and that satisfy

$$
L\left(\mathbf{F}_{r}\right) * L\left(\mathbf{F}_{r^{\prime}}\right)=L\left(\mathbf{F}_{r+r^{\prime}}\right) \quad\left(1<r, r^{\prime} \leq \infty\right)
$$

and

$$
L\left(\mathbf{F}_{r}\right)_{\gamma}=L\left(\mathbf{F}\left(1+\frac{r-1}{\gamma^{2}}\right)\right) \quad(1<r \leq \infty, 0<\gamma<\infty) .
$$

Moreover, it was seen that these two formulas imply that either all the $L\left(\mathbf{F}_{r}\right)$ are isomorphic to each other or that no two of them are isomorphic.

In this paper, we examine free products of general hyperfinite von Neumann algebras and express the answer in terms of the interpolated free group factors. (A finite von Neumann algebras is one which has an everywhere defined faithful trace-state; it is hyperfinite if it has a dense subalgebra which is the increasing union of finite dimensional algebras, i.e. for us "hyperfinite" means finite approximately finite dimensional; since by [5] there is only one hyperfinite $\mathrm{II}_{1}$-factor $R$, every hyperfinite von Neumann algebra is a direct sum of $R$ tensor an abelian algebra and $M_{n}$ tensor an abelian algebra, as $n$ ranges over $\mathbf{N} \backslash\{0\}$.) Our results are best organized using the concept of free dimension. A von Neumann algebra $\mathcal{A}$ belonging to a certain class of algebras and having specified faithful trace-state is assigned a positive real number as its free dimension, denoted fdim $(\mathcal{A})$. For example, fdim $\left(L\left(\mathbf{F}_{r}\right)\right)=r$, $\operatorname{fdim}(L(\mathbf{Z}))=\operatorname{fdim}(R)=1$, where $R$ is the hyperfinite $\mathrm{II}_{1}$ factor. Now suppose that $\mathcal{A}$ and $\mathcal{B}$ are hyperfinite von

Studies and research supported by the Fannie and John Hertz Foundation.

This work will form part of the author's Ph.D. thesis at the University of California, Berkeley.

Typeset by $\mathcal{A M}_{\mathcal{M}} \mathcal{S}-\mathrm{T}_{\mathrm{E}} \mathrm{X}$ 
Neumann algebras having specified faithful trace-state. We give precise conditions for $\mathcal{A} * \mathcal{B}$ to be a factor, (essentially, when $\mathcal{A}$ and $\mathcal{B}$ are not too "lumpy"), and show that when we have factoriality, $\mathcal{A} * \mathcal{B}=L(\mathbf{F}(\operatorname{fdim}(\mathcal{A})+\operatorname{fdim}(\mathcal{B})))$. When we do not have factoriality, we have $\mathcal{A} * \mathcal{B} \cong L\left(\mathbf{F}_{r}\right) \oplus C$, where $C$ is a finite dimensional algebra, and

$$
\operatorname{fdim}(\mathcal{A} * \mathcal{B})=\operatorname{fdim}(\mathcal{A})+\operatorname{fdim}(\mathcal{B})
$$

$C$ can be found by examining the minimal projections of $\mathcal{A}$ and $\mathcal{B}$, and $r$ is found using (3). Note that if the free group factors are isomorphic to each other, "free dimension" ceases to be well-defined, but also the need to find $r$ in the above expression disappears.

The proofs given here consist in large part of algebraic manipulations of the sort introduced in [4], but we also make use of the two pictures of $L\left(\mathbf{F}_{r}\right)$ contained in [9] and [4]. Both of these pictures depend on Voiculescu's random matrix model for freeness [14].

This paper has five sections. In $\S 1$ we establish notation and prove some theorems that will be our basic tools in the sequel; in $\S 2$ we examine free products of finite dimensional abelian algebras and related algebras; in $\S 3$ we examine free products of general finite dimensional algebras; in $\S 4$ we develop inductive limit techniques in order to extend the previous results to free products of general hyperfinite von Neumann algebras; in $\S 5$ we write down what these results imply for the free products of amenable groups, using Connes' charactarization in [2].

\section{$\S 1$. Notation and basic theorems.}

Throughout this paper, we will be working with finite von Neumann algebras, and each one will have a normalized, faithful trace associated to it. Moreover, when we write that two von Neumann algebras are isomorphic, (or one is contained in the other), we will mean that the isomorphism (inclusion) is trace-preserving. To save writing, we will not always describe these traces explicitly, but will rely on the conventions found in Remark 3.1 of [4]. In addition, we will use the following notation to specify the trace on a direct sum of algebras: for von Neumann algebras $A$ and $B$ with traces $\tau_{A}$ and $\tau_{B}, \underset{\alpha}{A} \oplus \underset{\beta}{B}$ where $\alpha, \beta \geq 0$ and $\alpha+\beta=1$, will denote the algebra $A \oplus B$ whose associated trace is $\tau(a, b)=\alpha \tau_{A}(a)+\beta \tau_{B}(b)$. In the case that one of the numbers $\alpha, \beta$ equals zero, say for example $\beta=0$, we will take $\underset{\alpha}{A} \oplus B_{\beta}$ to denote the algebra $A$ with trace $\tau_{A}$. If we also want to give names to the projections corresponding to the identity elements of $A$ and $B$, we will write

$$
\underset{\alpha}{A} \oplus \stackrel{q}{B},
$$

meaning $p=(1,0)$ and $q=(0,1)$. Similar notation will apply to direct sums of more that two algebras.

Traveling (and alternating) products will be as defined in 3.4 of [4], and here also $\Lambda(\ldots)$ will denote the set of all traveling products. Also, for an algebra $A, \stackrel{\circ}{A}$ will denote the kernel of the trace on $A$.

We will be using the interpolated free group factors [4,9], $L\left(\mathbf{F}_{r}\right)$ for $1<r<\infty$, and for convenience we will also let $L\left(\mathbf{F}_{1}\right)$ denote $L(\mathbf{Z})$.

Theorem 1.1. (Two projections.) Let $1>\alpha \geq \max \{\beta, 1-\beta\}>0$. Then

$$
(\stackrel{p}{\mathbf{C}} \oplus \underset{1-\alpha}{1-p} \underset{\mathbf{C}}{\mathbf{C}}) *(\underset{\beta}{q} \oplus \underset{1-\beta}{\mathbf{C}} \underset{\alpha+\beta}{\mathbf{C}}) \cong \underset{\alpha \wedge}{\stackrel{p}{\mathbf{C}}} \oplus(\underbrace{L^{\infty}\left(\left[0, \frac{\pi}{2}\right], \nu\right) \otimes M_{2}}_{2(1-\alpha)}) \oplus \underset{\alpha-\beta}{p \wedge(1-q)}
$$

where $\nu$ is a probability measure without atoms on $\left[0, \frac{\pi}{2}\right]$, and $L^{\infty}\left(\left[0, \frac{\pi}{2}\right], \nu\right)$ has trace given by integration with respect to $\nu$. In the picture of the right hand side of (4), we have

$$
\begin{aligned}
& p=1 \oplus\left(\begin{array}{ll}
1 & 0 \\
0 & 0
\end{array}\right) \oplus 1, \\
& q=1 \oplus\left(\begin{array}{cc}
\cos ^{2} \theta & \cos \theta \sin \theta \\
\cos \theta \sin \theta & \sin ^{2} \theta
\end{array}\right) \oplus 0,
\end{aligned}
$$


where $\theta \in\left[0, \frac{\pi}{2}\right]$.

Proof. The proof is the same as that of Proposition 3.2 of [4]. The algebra on the left hand side of (4) has a dense subalgebra which is a quotient of the universal unital $\mathrm{C}^{*}$-algebra generated by two projections. The trace on this subalgebra is then determined using Voiculescu's result [11] about the distribution of $p q p$. (We do the two cases $\beta \geq \frac{1}{2}$ and $\beta \leq \frac{1}{2}$ separately.)

Theorem 1.2. Let $A, B$ and $C$ be finite von Neumann algebras (with implicitly specified traces) and $n \geq 1$. Let

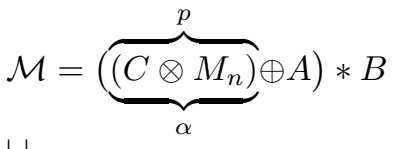

$$
\begin{aligned}
& \mathcal{N}=\left(\begin{array}{cc}
\stackrel{p}{M} & \oplus A) * B
\end{array}\right.
\end{aligned}
$$

and let $p_{1} \leq p$ be a minimal projection of $M_{n}$. Then in $p_{1} \mathcal{M} p_{1}$ we have that $p_{1} \mathcal{N} p_{1}$ and $C \otimes p_{1}$ are free, and together they generate $p_{1} \mathcal{M} p_{1}$, so

$$
p_{1} \mathcal{M} p_{1} \cong C * p_{1} \mathcal{N} p_{1} .
$$

Moreover, the central projection of $p_{1}$ in $\mathcal{M}$ equals the central projection of $p_{1}$ in $\mathcal{N}$.

Proof. For notational convenience, we identify $C$ with $(C \otimes 1) \oplus 0 \subset \mathcal{M}$. To see that $p_{1} \mathcal{N} p_{1}$ and $p_{1} C$ generate $p_{1} \mathcal{M} p_{1}$, note that $\mathcal{N}$ and $p_{1} C$ generate $\mathcal{M}$, so $\operatorname{span} \Lambda\left(\mathcal{N}, p_{1} C\right)$ is dense in $\mathcal{M}$ and $p_{1} \Lambda\left(\mathcal{N}, p_{1} C\right) p_{1}=\Lambda\left(p_{1} \mathcal{N} p_{1}, p_{1} C\right)$.

Now we shall show that a nontrivial alternating product in $\left(p_{1} \mathcal{N} p_{1}\right)^{o}$ and $p_{1} \stackrel{o}{C}$ has trace zero. Let $a=p_{1}-\frac{\alpha}{n}$. Then $\left(M_{n} \oplus A\right)^{o}=\mathbf{C} a+S$ where $S=\left\{s \in M_{n} \oplus A \mid \tau(s)=0, p_{1} s p_{1}=0\right\}$. So $\operatorname{span} \Lambda(\{a\} \cup S, \stackrel{o}{B})$ is a $*-$ algebra that is dense in $\mathcal{N}$. Let $x \in\left(p_{1} \mathcal{N} p_{1}\right)^{o}$. Then by the Kaplansky density theorem $x$ is the s.o.-limit of a bounded sequence $\left\{R_{k}\right\}_{k=1}^{\infty}$ in $\operatorname{span} \Lambda(\{a\} \cup S, \stackrel{\circ}{B})$. For $Q \in \operatorname{span}(\Lambda(\{a\} \cup S, \stackrel{o}{B}) \backslash\{1\})$, its easy to show that the trace of $p_{1} Q p_{1}$ is equal to a fixed constant times the coefficient of $a$ in $Q$. So since the trace of $R_{k}$ and the trace of $p_{1} R_{k} p_{1}$ tend to zero, we may assume that the coefficients in each $R_{k}$ of 1 and $a$ are zero. Since $R_{k}-p_{1} R_{k} p_{1}$ tends to zero, we may assume that also the coefficient of each element of $S$ in $R_{k}$ is zero, i.e. that each $R_{k} \in \operatorname{span}(\Lambda(\{a\} \cup S, \stackrel{o}{B}) \backslash(\{1, a\} \cup S))$. To prove the theorem it thus suffices to show that a nontrivial alternating product in $\Lambda(\{a\} \cup S, \stackrel{\circ}{B}) \backslash(\{1, a\} \cup S)$ and $p_{1} \stackrel{o}{C}$ has trace zero. But regrouping and multiplying some neighboring elements gives (a constant times) a nontrivial alternating product in $\{a\} \cup S \cup\left(p_{1} \stackrel{o}{C}\right)$ and $\stackrel{o}{B}$, which has trace zero by freeness.

Let $l$ be the central projection of $p_{1}$ in $\mathcal{N}$. Then $l \geq p$ and $l$ commutes with both $B$ and $M_{n} \oplus A$. Clearly the central projection of $p_{1}$ in $\mathcal{M}$ contains $l$. But as $l$ commutes with both $\left(C \otimes M_{n}\right) \oplus A$ and $B, l$ is in the center of $\mathcal{M}$, so the central projection of $p_{1}$ in $\mathcal{M}$ equals $l$.

By using these two theorems and paying attention to central projections, one can calculate the free products of direct sums of algebras, provided one knows the free products of the summands with various algebras. This idea is at the heart of much of what follows in this paper, but we get started using Rădulescu's picture of the interpolated free group factors [9] and some techniques that he developed.

Lemma 1.3. In a $W^{*}$-probability space $(\mathcal{C}, \psi)$ with $\psi$ a trace, let $\left\{a_{1}, a_{2}, c, u\right\}$ be $*$-free with $a_{1}$ and $a_{2}$ semicircular, $c$ circular and where u generates a diffuse abelian von Neumann algebra. Let $l \in\{u\}^{\prime \prime}$ be a self-adjoint projection having trace $t$, and let $v=\operatorname{pol}(l c l)$, a partial isometry from $l$ to $l$, such that $l c l=v\left(l c^{*} l c l\right)^{1 / 2}$. Consider $\mathcal{D}=$ $\left\{a_{2}, u, v^{*} a_{1} v, v^{*} c\right\}^{\prime \prime}$. Then $\mathcal{D}=L(\mathbf{F}(2+2 t))$.

Proof. We will show that, by adjusting $\mathcal{C}$ is necessary, we may assume that there exist semicircular elements $Z_{1}, Z_{2}$ and $Z_{3}$ in $\mathcal{C}$ such that $\left\{a_{2}, u, Z_{1}, Z_{2}, Z_{3}\right\}$ is a $*$-free family,

$$
\begin{aligned}
& v^{*} a_{1} v=l Z_{1} l, \\
& v^{*} c(1-l)=l Z_{2}(1-l), \\
& \left\{v^{*} c l\right\}^{\prime \prime}=\left\{l Z_{3} l\right\}^{\prime \prime} .
\end{aligned}
$$


If $t=1 / k$ for $k \in \mathbf{N} \backslash\{0\}$, we may apply Voiculescu's matrix model as Voiculescu did in Theorem 3.3 of [13] to find $Z_{1}, Z_{2}$ and $Z_{3}$ as required. For general $t$, let $k \in \mathbf{N}$ be such that $t \geq 1 / k$. Consider a $\mathrm{W}^{*}$-probability space $(\widetilde{\mathcal{C}}, \widetilde{\psi})$ in which there is a $*$-free family $\left\{\tilde{a}_{1}, \tilde{a}_{2}, \tilde{c}, \tilde{u}\right\}$ with $\tilde{a}_{1}$ and $\tilde{a}_{2}$ semicircular, $\tilde{c}$ circular, $\tilde{u}$ generating a diffuse abelian von Neumann algebra, and let $\tilde{l}, \tilde{p}$ be projections in $\{\tilde{u}\}^{\prime \prime}$ with $\tilde{l} \leq \tilde{p}$ and $\tilde{\psi}(\tilde{l})=\frac{1}{k}, \tilde{\psi}(\tilde{p})=\frac{1}{t k}$. Let $\tilde{v}=\operatorname{pol}(\tilde{l} \tilde{l} \tilde{l})$. We may now assume that there are semicircular $\widetilde{Z}_{1}, \widetilde{Z}_{2}$ and $\widetilde{Z}_{3}$ in $\widetilde{\mathcal{C}}$ such that $\left\{\tilde{a}_{2}, \tilde{u}, \widetilde{Z}_{1}, \widetilde{Z}_{2}, \widetilde{Z}_{3}\right\}$ is $*$-free and

$$
\begin{aligned}
& \tilde{v}^{*} \tilde{a}_{1} \tilde{v}=\tilde{l}_{\tilde{Z}} \tilde{l}_{1}, \\
& \tilde{v}^{*} \tilde{c}(1-\tilde{l})=\tilde{l} \widetilde{Z}_{2}(1-\tilde{l}), \\
& \left\{\tilde{v}^{*} \tilde{c}\right\}^{\prime \prime}=\left\{\tilde{l} \widetilde{Z}_{3} \tilde{l}^{\prime \prime} .\right.
\end{aligned}
$$

Let $a_{i}=\tilde{p} \tilde{a}_{i} \tilde{p}(i=1,2), c=\tilde{p} \tilde{c} \tilde{p}, u=\tilde{p} \tilde{u}$. By Proposition 2.3 of $[13],\left\{a_{1}, a_{2}, c, u\right\}$ is $*$-free in $\left(\tilde{p} \widetilde{\mathcal{C}} \tilde{p},\left.t k \widetilde{\psi}\right|_{\tilde{p} \tilde{p} \tilde{p}}\right)$. Letting $Z_{i}=\tilde{p} \widetilde{Z}_{i} \tilde{p}(i=1,2,3)$ we have also by the same proposition that in $\tilde{p} \widetilde{\mathcal{C}} \tilde{p}$ each $Z_{i}$ is semicircular and $\left\{a_{2}, u, Z_{1}, Z_{2}, Z_{3}\right\}$ is $*$-free. Writing $\tilde{l}=l$ (which in $\tilde{p} \tilde{\mathcal{C}} \tilde{p}$ has trace $t$ ), clearly (5) holds, as required.

So $\mathcal{D} \cong\left\{a_{2}, u, l Z_{1} l, l Z_{2}(1-l), l Z_{3} l\right\}^{\prime \prime}$, which by Rădulescu's definition of the interpolated free group factors [9] is $L(\mathbf{F}(2+2 t))$.

Lemma 1.4. For $0 \leq \delta \leq 1$ we have

$$
L(\mathbf{Z}) * \underset{\delta}{L(\mathbf{Z})} \oplus \underset{1-\delta}{\mathbf{C}})=L\left(\mathbf{F}\left(1+\delta^{2}+2 \delta(1-\delta)\right)\right) .
$$

Proof. Let us first prove the case $\delta \geq 1 / 2$. Let $\mathcal{A}$ denote the algebra of the left hand side of (6), and let $\tau$ be its trace. We can model $\mathcal{A}$ as follows. Let $(\mathcal{M}, \tau)=\left(\mathcal{N} \otimes M_{2}, \phi \otimes \tau_{2}\right)$, where $(\mathcal{N}, \phi)$ is a $\mathrm{W}^{*}$-probability space containing enough free elements (to be specified below), $\phi$ is a trace and $\tau_{2}$ is the normalized trace on $M_{2}$. In $\mathcal{M}$ let $X=\left(\begin{array}{ll}a_{1} & c \\ c^{*} & a_{2}\end{array}\right)$, $Y=\left(\begin{array}{ll}0 & 0 \\ 0 & u\end{array}\right)$, where in $(\mathcal{N}, \phi), a_{1}$ and $a_{2}$ are semicircular, $c$ is circular, $\mathrm{u}$ is a Haar unitary and $\left\{a_{1}, a_{2}, c, u\right\}$ is $*$-free. Let $l \in\{u\}^{\prime \prime}$ in $\mathcal{N}$ be a self-adjoint projection of trace $\frac{1}{\delta}-1$. Let $p=\left(\begin{array}{ll}l & 0 \\ 0 & 1\end{array}\right)$, so $\tau(p)=(2 \delta)^{-1}$. In $\left(p \mathcal{M} p,\left.2 \delta \tau\right|_{p \mathcal{M} p}\right)$ consider the elements $p X p$ and $p Y p$. By 2.3 of [13], they are $*$-free and $p X p$ is semicircular. Clearly $p Y p$ generates

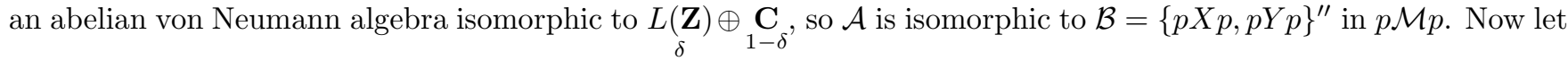

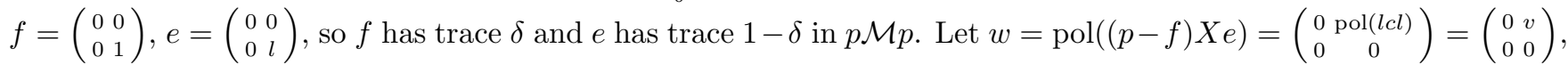
(where $v=\operatorname{pol}(l c l)$ ), a partial isometry from $e$ to $p-f$. Then $f \mathcal{B} f=\left\{f X f, f Y f, w^{*} X w, w^{*} X f\right\}^{\prime \prime}$, which is clearly isomorphic to the von Neumann algebra $\left\{a_{2}, u, v^{*} a_{1} v, v^{*} c\right\}^{\prime \prime}$ in $\mathcal{N}$. Lemma 1.3 gives us that $\mathcal{A}_{\delta} \cong f \mathcal{B} f=\mathrm{L}(\mathbf{F}(2 / \delta))$, and formula (2) gives $\mathcal{A}=L\left(\mathbf{F}\left(1+\delta^{2}+2 \delta(1-\delta)\right)\right)$ as required.

Let us prove the general case by induction on $k$, where $\frac{1}{k+1} \leq \delta \leq \frac{1}{k}$. We just proved the case $k=1$. For $k \geq 2$, let

$$
\begin{aligned}
& \mathcal{M}=(L(\mathbf{Z}) \oplus L(\mathbf{Z})) *(L(\mathbf{Z}) \oplus \mathbf{C}) \\
& \cup \\
& \mathcal{N}_{2}=(L(\mathbf{Z}) \oplus L(\mathbf{Z})) *(\mathbf{C} \oplus \mathbf{C}) \\
& \cup \\
& \mathcal{N}_{1}=(\mathbf{C} \oplus L(\mathbf{Z})) *(\mathbf{C} \oplus \mathbf{C}) \\
& \cup \\
& \mathcal{N}_{0}=(\underset{\delta}{\stackrel{1-p}{\mathbf{C}}} \oplus \underset{1-\delta}{\stackrel{p}{\mathbf{C}}}) *(\underset{\delta}{\stackrel{1-p}{\mathbf{C}}} \oplus \underset{1-\delta}{\stackrel{q}{\mathbf{C}}}) .
\end{aligned}
$$

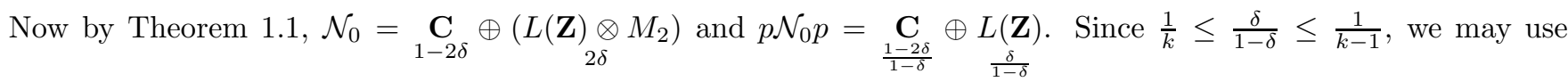
Theorem 1.2 and inductive hypothesis to conclude that $p \mathcal{N}_{1} p=L\left(\mathbf{F}\left(1+(1+\delta)^{2}\left(\delta^{2}+2 \delta(1-2 \delta)\right)\right)\right)$. Since the central projection of $p$ in $\mathcal{N}_{0}$ is 1 , we conclude by 1.2 and (2) that $\mathcal{N}_{1}=L\left(\mathbf{F}\left(1+2 \delta-3 \delta^{2}\right)\right)$. Applying Theorem 1.2 twice again, we get $\mathcal{N}_{2}=L\left(\mathbf{F}\left(1+2 \delta-2 \delta^{2}\right)\right)$ and $\mathcal{M}=L\left(\mathbf{F}\left(1+2 \delta-\delta^{2}\right)\right)$. 
Remark 1.5. We now have another description of the interpolated free group factors $L\left(\mathbf{F}_{r}\right)(r \geq 1)$ by writing

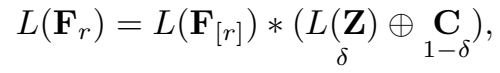

where $[r]$ is the integer part of $r$ and $\delta^{2}+2 \delta(1-\delta)=r-[r], 0 \leq \delta<1$. Thus $L\left(\mathbf{F}_{r}\right)=\mathrm{E}(\mathbf{Z}) * D_{r-1}$ where $D_{r-1}$

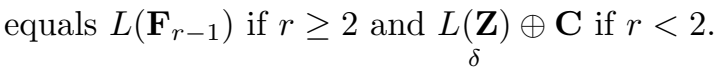

Lemma 1.6. For $s \geq 1$ and $0 \leq \delta \leq 1$ we have

$$
\left.L(\mathbf{Z}) * \underset{\delta}{L\left(\mathbf{F}_{s}\right)} \oplus \underset{1-\delta}{\mathbf{C}}\right)=L\left(\mathbf{F}\left(1+s \delta^{2}+2 \delta(1-\delta)\right)\right) .
$$

Proof. First show that $L(\mathbf{Z}) * \underset{\alpha}{\mathbf{C}} \oplus \mathbf{C})=L(\mathbf{F}(1+2 \alpha(1-\alpha)))$ by taking $\alpha \geq 1 / 2$ and writing

$$
\begin{aligned}
& \mathcal{M}=\underset{\alpha}{L(\mathbf{Z})} \oplus L(\mathbf{Z})) * \underset{\alpha}{\mathbf{C} \oplus \mathbf{C})} \\
& \mathcal{N}_{1}=(\mathbf{C} \oplus L(\mathbf{Z})) *(\mathbf{C} \oplus \mathbf{C})
\end{aligned}
$$

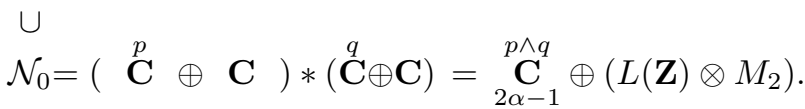

Now apply 1.2 twice, using 1.4 to find $\mathcal{N}_{1}$. One then finds (7) by applying 1.2 again.

Proposition 1.7. For $r, s \geq 1$ and $0 \leq \gamma, \delta \leq 1$ we have

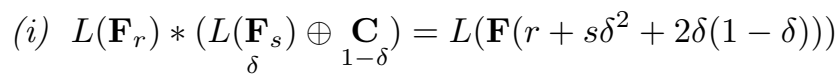

$$
\text { and (ii) }\left(\underset{\gamma}{\left(\mathbf{F}_{r}\right)} \oplus \underset{1-\gamma}{\mathbf{C}}\right) *\left(L \underset{\delta}{\left(\mathbf{F}_{s}\right)} \oplus \underset{1-\delta}{\mathbf{C}}\right)= \begin{cases}L\left(\mathbf{F}\left(r \gamma^{2}+2 \gamma(1-\gamma)+s \delta^{2}+2 \delta(1-\delta)\right)\right) & \text { if } \gamma+\delta \geq 1 \\ \underbrace{L\left(\mathbf{F}\left((\gamma+\delta)^{-2}\left(r \gamma^{2}+s \delta^{2}+4 \gamma \delta\right)\right)\right.}_{\gamma+\delta} \oplus_{1-\gamma-\delta}^{\mathbf{C}} & \text { if } \gamma+\delta \leq 1 .\end{cases}
$$

Proof. 1.6 gives (i) for the case $r=1$. Now we prove (ii) for the case $r=s=1$. Take

$$
\begin{aligned}
& \mathcal{M}=(L(\mathbf{Z}) \oplus \mathbf{C}) *(L(\mathbf{Z}) \oplus \mathbf{C}) \\
& \cup \\
& \mathcal{N}_{1}=(\mathbf{C} \oplus \mathbf{C}) *(L(\mathbf{Z}) \oplus \mathbf{C}) \\
& \cup \\
& \mathcal{N}_{0}=\left(\underset{\gamma}{\mathbf{C}} \oplus_{1-\gamma}^{\mathbf{C}}\right) *\left(\underset{\delta}{\mathbf{C}} \oplus_{1-\delta}^{\mathbf{C}}\right),
\end{aligned}
$$

find $\mathcal{N}_{0}$ using Theorem 1.1 and apply Theorem 1.2 twice (as we did in 1.4) to find $\mathcal{N}_{1}$, then $\mathcal{M}$. The cases $\gamma \geq$ $\max (\delta, 1-\delta)$ and $\gamma \leq \max (\delta, 1-\delta)$ are done separately, each making use of 1.6.

Now we can prove (i) for all $r, s \geq 1$. Let $L\left(\mathbf{F}_{r}\right)=L\left(\mathbf{F}_{[r]}\right) * D_{r-1}$, where $D_{r-1}$ is as in Remark 1.5. Then $L\left(\mathbf{F}_{r}\right) *\left(\underset{\delta}{\left(\mathbf{F}_{s}\right)} \oplus \underset{1-\delta}{\mathbf{C}}\right)=D_{r-1} * L\left(\mathbf{F}\left(1+s \delta^{2}+2 \delta(1-\delta)\right)\right.$ by 1.6. If $r \geq 2$ we are done, and if $s \delta^{2}+2 \delta(1-\delta) \geq 1$

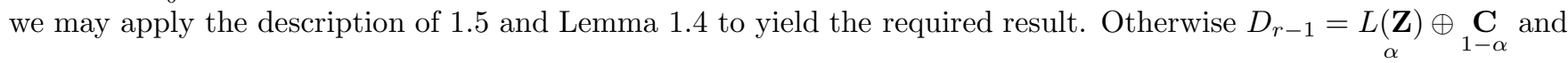

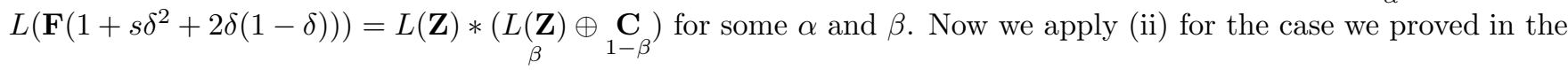
preceding paragraph.

Having (i) for all $r, s \geq 1$, we may now prove (ii) for all $r, s \geq 1$ using the same argument we did earlier for the case $r=s=1$. 
Remark 1.8. Knowing 1.5 and 1.7, it is now easy to show that for any $A$, if $L(\mathbf{Z}) * A=L\left(\mathbf{F}_{1+s}\right)$ for some $s \geq 0$, then $L\left(\mathbf{F}_{r}\right) * A=L\left(\mathbf{F}_{r+s}\right)$ for all $r \geq 1$.

\section{$\S 2$. Finite dimensional abelian algebras.}

Definition 2.1. The free dimension of the algebra

$$
A=\underset{\alpha_{1}}{\mathbf{C}} \oplus \cdots \oplus \underset{\alpha_{n}}{\mathbf{C}} \quad(n \geq 1)
$$

is

$$
\operatorname{fdim}(A)=\sum_{\substack{1 \leq i, j \leq n \\ i \neq j}} \alpha_{i} \alpha_{j} .
$$

This includes the cases $\operatorname{fdim}(\mathbf{C})=0$ and $\operatorname{fdim}(\underset{\alpha}{\mathbf{C}} \oplus \underset{1-\alpha}{\mathbf{C}})=2 \alpha(1-\alpha)$. We also define the free dimension of

$$
B=\underset{\alpha_{0}}{L\left(\mathbf{F}_{s}\right)} \oplus \underset{\alpha_{1}}{\mathbf{C}} \oplus \cdots \oplus \underset{\alpha_{n}}{\mathbf{C}} \quad(s \geq 1, n \geq 0)
$$

to be

$$
\operatorname{fdim}(B)=s \alpha_{0}^{2}+\sum_{\substack{0 \leq i, j \leq n \\ i \neq j}} \alpha_{i} \alpha_{j} .
$$

This includes the special case $\operatorname{fdim}\left(L\left(\mathbf{F}_{s}\right)\right)=s$.

Of course if the free group factors are isomorphic to each other then the free dimension of the algebra of (8) can take on a continuum of values, hence is non-unique. We will sometimes want to let $s$ in (8) be determined by the requirement that $B$ have a certain free dimension, but in the case of non-uniqueness of free dimension this is no problem since then all values of $s$ would give the same algebra.

In this section we will show the addition formula (3) for free products of algebras as in 2.1 and also decide in which cases we have a factor. In particular, for finite dimensional abelian algebras $A$ and $B$ we have that $A * B$ is a factor if and only if $\operatorname{dim} A, \operatorname{dim} B \geq 2, \operatorname{dim} A+\operatorname{dim} B \geq 5$ and the largest mass of an atom in $A$ plus the largest mass of an atom in $B$ is $\leq 1$. Our proofs will consist of induction arguments using the results of the last section. The details are fairly tedious and elementary, so we will only give outlines of the proofs.

Lemma 2.2. Let

$$
A=L \underset{\alpha_{0}}{L\left(\mathbf{F}_{r}\right)} \stackrel{p_{1}}{\mathbf{C}} \oplus \cdots \oplus \underset{\alpha_{1}}{p_{n}} \underset{\alpha_{n}}{\mathbf{C}},
$$

where $n \geq 0, \alpha_{0}>0, \alpha_{1} \geq \alpha_{2} \geq \cdots \alpha_{n}>0$ and $r \geq 1$ Let $\frac{1}{2} \leq \beta \leq 1$. Then

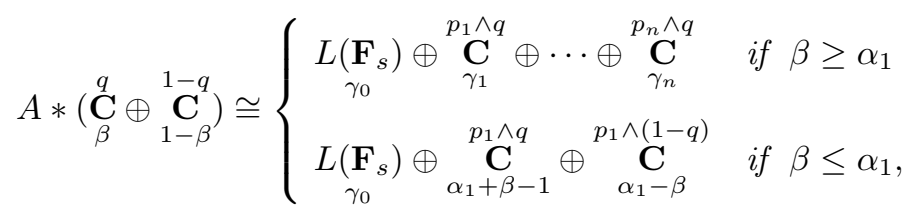

where $\gamma_{i}=\max \left(\beta+\alpha_{i}-1,0\right)$ for $1 \leq i \leq n$ and where $s$ is such that the free dimension of the right hand side of (9) equals $\operatorname{fdim}(A)+2 \beta(1-\beta)$.

Proof. For $n=0$, by Remark 1.8 we need only do the case $r=1$. But this was done in the proof of 1.6 . The case $\mathrm{n}=1$ is similarly proved using 1.2 and 1.7. One then proceeds by induction on $n$, proving the inductive step by writing

$$
\begin{aligned}
& \left.\mathcal{M}=\left(\underset{\alpha_{0}}{\left(L \mathbf{F}_{r}\right.}\right) \oplus \underset{\alpha_{1}}{\mathbf{C}} \oplus \underset{\alpha_{2}}{\mathbf{C}} \oplus \underset{\alpha_{3}}{\mathbf{C}} \oplus \cdots \oplus \underset{\alpha_{n}}{\mathbf{C}}\right) *(\underset{\beta}{\mathbf{C}} \oplus \underset{1-\beta}{\mathbf{C}}) \\
& \mathcal{N}=\left(L\left(\mathbf{F}_{\alpha_{0}}\right) \oplus \underset{\alpha_{1}+\alpha_{2}}{\mathbf{C}} \oplus \underset{\alpha_{3}}{\mathbf{C}} \oplus \cdots \oplus \underset{\alpha_{n}}{\mathbf{C}}\right) *(\underset{\beta}{\mathbf{C}} \oplus \underset{1-\beta}{\mathbf{C}})
\end{aligned}
$$

and using 1.2 and 1.7. Note that the case $n=2$ and $\alpha_{1}+\alpha_{2} \geq \beta$ requires an additional induction argument on $k$ such that $(k-1) \alpha_{0} \leq \alpha_{2} \leq k \alpha_{0}$. 
Theorem 2.3. Let

$$
A=\underset{\alpha_{1}}{\mathbf{C}} \oplus \cdots \oplus \underset{\alpha_{n}}{p_{n}}, \quad B=\underset{\beta_{1}}{\mathbf{q _ { 1 }}} \oplus \cdots \oplus \underset{\beta_{m}}{\mathbf{q}}
$$

be finite dimensional abelian algebras, where each $\alpha_{i}$ and $\beta_{j}>0, n, m \geq 2$ and $n+m \geq 5$. Then

$$
A * B \cong L\left(\mathbf{F}_{s}\right) \oplus \bigoplus_{\substack{1 \leq i \leq n \\ 1 \leq j \leq m}} \stackrel{p_{i j} \wedge q_{j}}{\mathbf{C}},
$$

where $\gamma_{i j}=\max \left(\alpha_{i}+\beta_{j}-1,0\right)$ and where $s$ is chosen so that $\operatorname{fdim}(A * B)=\operatorname{fdim}(A)+\operatorname{fdim}(B)$.

Proof. Proceed by induction on $n+m$. Assume $n \geq 3$ and prove the initial case $n+m=5$ as well as the inductive step by writing

$$
\begin{aligned}
& \mathcal{M}=\left(\underset{\alpha_{1}}{\mathbf{C}} \oplus \underset{\alpha_{2}}{\mathbf{C}} \oplus \underset{\alpha_{3}}{\mathbf{C}} \oplus \cdots \oplus \underset{\alpha_{n}}{\mathbf{C}}\right) *\left(\underset{\beta_{1}}{\mathbf{C}} \oplus \cdots \oplus \underset{\beta_{m}}{\mathbf{C}}\right) \\
& \cup \\
& \mathcal{N}=\left(\underset{\alpha_{1}+\alpha_{2}}{\mathbf{C}} \underset{\alpha_{3}}{\mathbf{C}} \oplus \cdots \oplus \underset{\alpha_{n}}{\mathbf{C}}\right) *\left(\underset{\beta_{1}}{\mathbf{C}} \oplus \cdots \oplus \underset{\beta_{m}}{\mathbf{C}}\right),
\end{aligned}
$$

and applying 1.2 using 2.2 .

It will be useful to have also the following proposition, which is straightforwardly proved from the above results.

Proposition 2.4. Let

$$
\begin{aligned}
& A=L \underset{\alpha_{0}}{L\left(\mathbf{F}_{r}\right)} \oplus \underset{\alpha_{1}}{\mathbf{p}_{1}} \oplus \cdots \oplus \underset{\alpha_{n}}{\mathbf{C}}\left(n \geq 0, r \geq 1, \alpha_{0} \geq 0\right) \\
& B=L \underset{\beta}{L\left(\mathbf{F}_{s}\right)} \oplus \underset{\beta_{1}}{\mathbf{q _ { 1 }}} \oplus \cdots \oplus \underset{\beta_{m}}{\mathbf{q _ { m }}}\left(m \geq 0, s \geq 1, \beta_{0} \geq 0\right),
\end{aligned}
$$

where $\alpha_{0}+\beta_{0}>0$. Then

$$
A * B \cong L\left(\mathbf{F}_{t}\right) \oplus \bigoplus_{\substack{1 \leq i \leq n \\ 1 \leq j \leq m}} \stackrel{p_{i} \wedge q_{j}}{\gamma_{i j}},
$$

where $\gamma_{i j}=\max \left(\alpha_{i}+\beta_{j}-1,0\right)$ and where $t$ is chosen so that $\operatorname{fdim}(A * B)=\operatorname{fdim}(A)+\operatorname{fdim}(B)$.

\section{$\S 3$. Finite dimensional algebras.}

In this section we determine the free product of any two finite dimensional algebras. $\mathbf{Z}_{n}$ will denote the cyclic group of order $n, M_{n}$ the $n \times n$ complex matrices and for a (finite) factor $\mathcal{M}$ and $0<t \leq 1, \mathcal{M}_{t}$ denotes an algebra isomorphic to $p \mathcal{M} p$, where $p \in \mathcal{M}$ is a self-adjoint projection of trace $t$.

Proposition 3.1. Let $\mathcal{A}$ be a finite von Neumann algebra such that $\mathcal{N}=L\left(\mathbf{Z}_{n}\right) * \mathcal{A}$ is a factor. Then $\mathcal{M}=M_{n} * \mathcal{A}$ is a factor and $\mathcal{M}_{\frac{1}{n}} \cong\left(\mathcal{N}_{\frac{1}{n}}\right) * L\left(\mathbf{F}_{n-1}\right)$.

Proof. Let $p_{1}, \ldots, p_{n}$ be an orthogonal family of minimal projections of $M_{n}$, and let $u=\sum_{1 \leq j \leq n} e^{2 \pi i j / n} p_{j}$. Then $\operatorname{span} \Lambda\left(\left\{u, u^{2}, \ldots, u^{n-1}\right\}, \stackrel{o}{\mathcal{A}}\right)$ is a dense $*-$ subalgebra of $\mathcal{N}$. Since $\mathcal{N}$ is a factor, there are partial isometries $x_{k} \in \mathcal{N}$ from $p_{k}$ to $p_{1}$, i.e. such that $x_{k}^{*} x_{k}=p_{k}, x_{k} x_{k}^{*}=p_{1}(2 \leq k \leq n)$. Let $v_{k} \in M_{n}(2 \leq k \leq n)$ be partial isometries from $p_{k}$ to $p_{1}$. Then $p_{1} \mathcal{M} p_{1}$ is generated by $p_{1} \mathcal{N} p_{1}$ together with $\left\{v_{k} x_{k}^{*} \mid 2 \leq k \leq n\right\}$.

Claim 3.1a. Each $v_{k} x_{k}^{*}$ is a Haar unitary in $p_{1} \mathcal{M} p_{1}$.

Indeed. It is clearly a unitary, we need only show that the trace of a positive power is zero. We show that a nontrivial alternating product in $v_{k}$ and $x_{k}^{*}$ has trace zero. Now since the trace of $x_{k}^{*}$ is zero and $x_{k}^{*} u=e^{2 \pi i / n} x_{k}^{*}$, we may approximate $x_{k}^{*}$ in strong-operator topology by a bounded sequence in $\operatorname{span}\left(\Lambda\left(\left\{u, u^{2}, \ldots, u^{n-1}\right\}, \mathcal{A}\right) \backslash\left\{1, u, u^{2}, \ldots, u^{n-1}\right\}\right)$. Hence it suffices to show that a nontrivial alternating product in $\left\{v_{k}\right\}$ and $\Lambda\left(\left\{u, u^{2}, \ldots, u^{n-1}\right\}, \stackrel{o}{\mathcal{A}}\right) \backslash\left\{1, u, u^{2}, \ldots, u^{n-1}\right\}$ has trace zero. But regrouping and multiplying some neighbors gives (a constant times) a nontrivial alternating product in $\left\{v_{k}, u, u^{2}, \ldots, u^{n-1}\right\}$ and $\stackrel{o}{\mathcal{A}}$, which by freeness has trace zero. This proves the claim. 
Claim 3.1b. $\left\{p_{1} \mathcal{N} p_{1},\left(v_{k} x_{k}^{*}\right)_{2 \leq k \leq n}\right\}$ is $*$-free.

Indeed. We need only show that a nontrivial traveling product in $\left(p_{1} \mathcal{N} p_{1}\right)^{o},\left\{\left(v_{2} x_{2}^{*}\right)^{m} \mid m \in \mathbf{Z} \backslash\{0\}\right\}, \ldots,\left\{\left(v_{n} x_{n}^{*}\right)^{m} \mid\right.$ $m \in \mathbf{Z} \backslash\{0\}\}$ has trace zero. Regrouping gives a nontrivial alternating product in

$$
\left(p_{1} \mathcal{N} p_{1}\right)^{o} \cup \bigcup_{2 \leq k \leq n}\left(\left(p_{1} \mathcal{N} p_{1}\right) x_{k} \cup x_{k}^{*}\left(p_{1} \mathcal{N} p_{1}\right)\right) \cup \bigcup_{\substack{2 \leq i, j \leq n \\ i \neq j}} x_{i}^{*}\left(p_{1} \mathcal{N} p_{1}\right) x_{j}
$$

and

$$
\left\{v_{k}, v_{k}^{*} \mid 2 \leq k \leq n\right\} \cup\left\{v_{i}^{*} v_{j} \mid 2 \leq i, j \leq n, i \neq j\right\} .
$$

Just as above, we may use the Kaplansky density theorem to show that each element of the set (10) is the limit in strong-operator topology of a bounded sequence in $\operatorname{span}\left(\Lambda\left(\left\{u, u^{2}, \ldots, u^{n-1}\right\}, \stackrel{o}{\mathcal{A}}\right) \backslash\left\{1, u, u^{2}, \ldots, u^{n-1}\right\}\right)$. So it suffices to show that a nontrivial alternating product in $\Lambda\left(\left\{u, u^{2}, \ldots, u^{n-1}\right\}, \mathcal{A}\right) \backslash\left\{1, u, u^{2}, \ldots, u^{n-1}\right\}$ and the set (11) has trace zero. Regrouping and multiplying some neighbors gives (a constant times) a nontrivial traveling product in $\left\{v_{j}, v_{j}^{*} \mid 2 \leq j \leq n\right\} \cup\left\{u, u^{2}, \ldots, u^{n-1}\right\}$ and $\stackrel{o}{\mathcal{A}}$, which by freeness has trace zero. This proves the claim and hence the proposition.

The above proposition, together with Theorem 1.2 and the results of $\S 2$ allows us to determine the free product of finite dimensional algebras $A$ and $B$, provided that the largest trace of a minimal projection of $A$ plus the largest trace of a minimal projection of $B$ is $\leq 1$. However, we still cannot say what, for example, $\left.M_{2} * \underset{2 / 3}{\mathbf{C}} \oplus \underset{1 / 3}{\mathbf{C}}\right)$ is. Hence the following:

Proposition 3.2. Let $n \geq 2, \frac{1}{2} \leq \alpha<1$. Then

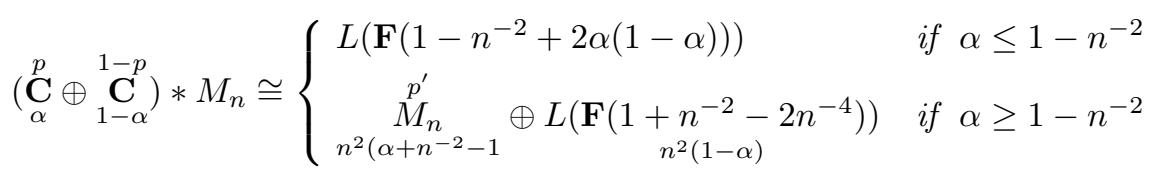

and $p^{\prime} \leq p$.

Proof. Let $\mathcal{M}$ be the left hand side of (12). If $\alpha \leq 1-n^{-1}$ then the result follows immediately from Theorem 2.3 and Proposition 3.1, so suppose $\alpha>1-n^{-1}$. Let $\left(q_{i}\right)_{1 \leq i \leq n}$ be an orthogonal family of minimal projections of $M_{n}$ and let $\mathcal{N}_{1}=\left(\{p\} \cup\left\{q_{i} \mid 1 \leq i \leq n\right\}\right)^{\prime \prime}$. Also let $\left\{q_{i} \mid 1 \leq i \leq n\right\} \cup\left\{v_{i j} \mid 1 \leq i, j \leq n, i \neq j\right\}$ be a system of matrix units in $M_{n}$, where $v_{i j}$ is a partial isometry from $q_{j}$ to $q_{i}$. By Theorem 2.3,

$$
\mathcal{N}_{1} \cong\left\{\begin{array}{cl}
\stackrel{p \wedge q_{1}}{\mathbf{C}} \oplus \cdots \oplus \underset{\alpha+n^{-1}-1}{\stackrel{p \wedge q_{n}}{\mathbf{C}}} \oplus L\left(\mathbf{F}\left(1+\frac{n-2}{n^{2}}\right)\right) & \text { if } n \geq 3 \\
\alpha+n^{-1}-1 & \text { if } n=2 . \\
\underset{\alpha-\frac{1}{2}}{\mathbf{C}} \oplus \underset{\alpha-\frac{1}{2}}{\mathbf{C}} \oplus \underset{\substack{h(1-\alpha) \\
2(1-\alpha)}}{\left.(\mathbf{Z}) \otimes M_{2}\right)} &
\end{array}\right.
$$

Then $q_{i}=p \wedge q_{i}+h_{i}$ for $h_{i} \leq h$ and $h=h_{1}+\cdots+h_{n}, \tau\left(h_{i}\right)=1-\alpha$.

$\mathcal{M}$ is generated by $\mathcal{N}_{1}$ together with $\left\{v_{1 j} \mid 2 \leq j \leq n\right\}$, so $q_{1} \mathcal{M} q_{1}$ is generated by $\left\{v_{1 i} h \mathcal{N}_{1} h v_{j 1} \mid 1 \leq i, j \leq n\right\}$ together with $\left\{v_{1 j}\left(p \wedge q_{j}\right) v_{j 1} \mid 2 \leq j \leq n\right\}$. Note that $v_{1 j}\left(p \wedge q_{j}\right) v_{j 1}=q_{1}-v_{1 j} h_{j} v_{j 1}$. Let $y_{1 j} \in h \mathcal{N}_{1} h$ be a partial isometry from $h_{1}$ to $v_{1 j} h_{j} v_{j 1}$. Then $v_{1 j} y_{j 1}$ is a partial isometry from $h_{1}$ to $v_{1 j} h_{j} v_{j 1}$ and $q_{1} \mathcal{M} q_{1}$ is generated by $h_{1} \mathcal{N}_{1} h_{1}$ together with $\left\{v_{1 j} y_{j 1} \mid 2 \leq j \leq n\right\}$. 
Claim 3.2a. $\left\{q_{1} \mathcal{N}_{1} q_{1},\left(\left\{v_{1 j} h_{j} v_{j 1}\right\}\right)_{2 \leq j \leq n}\right\}$ is free in $q_{1} \mathcal{M} q_{1}$.

Indeed. Let $c_{j}=v_{1 j}\left(h_{j}-n(1-\alpha) q_{j}\right) v_{j 1}$. We need only show that a nontrivial traveling product in $\left(q_{1} \mathcal{N}_{1} q_{1}\right)^{o}$, $\left(\left\{c_{j}\right\}\right)_{2 \leq j \leq n}$ has trace zero. Let $a=p-\alpha, b_{j}=q_{j}-n^{-1}(1 \leq j \leq n)$. Then $\operatorname{span} \Lambda\left(\{a\},\left\{b_{j} \mid 1 \leq j \leq n\right\}\right)$ is a dense $*$-subalgebra of $\mathcal{N}_{1}$. If $x \in \mathcal{N}_{1}, \tau(x)=0$ and $q_{j} x=x$ for some $1 \leq j \leq n$, then, as one can easily show, $x$ is the s.o.-limit of a bounded sequence in $\operatorname{span}\left(\Lambda\left(\{a\},\left\{b_{j} \mid 1 \leq j \leq n\right\}\right) \backslash\left\{1, b_{1}, \ldots, b_{n}\right\}\right)$. So in particular, each $x \in\left(q_{1} \mathcal{N}_{1} q_{1}\right)^{\circ}$ and each $h_{j}-n(1-\alpha) q_{j}(2 \leq j \leq n)$ is the limit of such a sequence. Thus we must only show that a nontrivial alternating product in $\Lambda\left(\{a\},\left\{b_{j} \mid 1 \leq j \leq n\right\}\right) \backslash\left\{1, b_{1}, \ldots, b_{n}\right\}$ and $\left\{v_{1 j}, v_{j 1} \mid 2 \leq j \leq n\right\}$ has trace zero. Regrouping gives a nontrivial alternating product in $\{a\}$ and $\left\{b_{1}, \ldots, b_{n}\right\} \cup\left\{v_{1 j}, v_{j 1} \mid 2 \leq j \leq n\right\}$, which by freeness has trace zero. This proves Claim 3.2a.

Now $q_{1} \mathcal{M} q_{1}$ is generated by $\mathcal{N}_{2}$ together with $\left\{v_{1 j} y_{j 1} \mid 2 \leq j \leq n\right\}$, where we let

$$
\mathcal{N}_{2}=\left(q_{1} \mathcal{N}_{1} q_{1} \cup\left\{v_{1 j} h_{j} v_{j 1} \mid 2 \leq j \leq n\right\}\right)^{\prime \prime}
$$

(with $q_{1}$ for identity element). But

$$
q_{1} \mathcal{N}_{1} q_{1}=\underset{1+n \alpha-n}{\stackrel{p_{1} \wedge q}{\mathbf{C}}} \oplus \underset{n(1-\alpha)}{L\left(\mathbf{F}_{n-1}\right)},
$$

so by the above claim we have

$$
\left.\mathcal{N}_{2} \cong \underset{n(1-\alpha)}{\left(L\left(\mathbf{F}_{n-1}^{h_{1}}\right)\right.} \oplus \stackrel{p_{1} \wedge q}{\mathbf{C}}\right) *(\underset{n(1-\alpha)}{v_{12} h_{2} v_{21}} \overbrace{}^{v_{12}\left(q_{2} \wedge p\right) v_{21}} \mathbf{C}) * \cdots *\left(\underset{n(1-\alpha)}{v_{1 n} h_{n} v_{n 1}} \oplus^{v_{1 n}\left(q_{n} \wedge p\right) v_{n 1}} \mathbf{C}\right)
$$

Applying 2.2 gives

Case 1. $\alpha \leq 1-n^{-2}$,

. then

$$
\mathcal{N}_{2}=L\left(\mathbf{F}\left(n^{2}\left(1-n+2 n \alpha-(1+n) \alpha^{2}\right)\right)\right),
$$

Case 2. $\alpha \geq 1-n^{-2}$,

. then

$$
\mathcal{N}_{2}=L\left(\mathbf{F}\left(2-n^{-1}-n^{-2}\right)\right) \oplus \stackrel{\left(p \wedge q_{1}\right) \wedge\left(v_{12}\left(p \wedge q_{2}\right) v_{21}\right) \wedge \cdots \wedge\left(v_{1 n}\left(p \wedge q_{n}\right) v_{n 1}\right)}{\underset{1-n^{2}(1-\alpha)}{\mathbf{C}}(1-\alpha)}
$$

so $h_{1} \leq k, v_{1 j} h_{j} v_{j 1} \leq k(2 \leq j \leq n)$.

We will compute $h_{1} \mathcal{M} h_{1}$ as a way of determining $q_{1} \mathcal{M} q_{1}=\left(q_{1} \mathcal{N}_{2} q_{1} \cup\left\{v_{1 j} y_{j 1} \mid 2 \leq j \leq n\right\}\right)^{\prime \prime}$ and hence $\mathcal{M}=\left(q_{1} \mathcal{M} q_{1}\right) \otimes M_{n}$. Let $z_{j} \in\left\{h_{1}, v_{1 j} h_{j} v_{j 1}\right\}^{\prime \prime}$ (the double commutant taken having $q_{1}$ for identity) be a partial isometry from $v_{1 j} h_{j} v_{j 1}$ to $h_{1},(2 \leq j \leq n)$. Then $h_{1} \mathcal{M} h_{1}$ is generated by $h_{1} \mathcal{N}_{2} h_{1}$ together with $\left\{z_{j} v_{1 j} y_{j 1} \mid 2 \leq j \leq n\right\}$.

Claim 3.2b. $z_{j} v_{1 j} y_{j 1}$ is a Haar unitary in $h_{1} \mathcal{M} h_{1}$ for $2 \leq j \leq n$.

Indeed. Let $d=h_{1}-n(1-\alpha) q_{1}, c_{j}=v_{1 j} \tilde{c}_{j} v_{j 1}$ where $\tilde{c}_{j}=h_{j}-n(1-\alpha) q_{j}(2 \leq j \leq n)$. Then $z_{j}$ is the s.o.-limit of a bounded sequence in $\operatorname{span} \Lambda\left(\{d\},\left\{c_{j}\right\}\right)$. Hence it suffices to show that a nontrivial alternating product of length $2 m>0$ in $\Lambda\left(\{d\},\left\{c_{j}\right\}\right)$ and $v_{1 j} y_{j 1}$ has trace zero. Noting that $\tilde{c}_{j}$ and $y_{j 1}$ lie in $\mathcal{N}_{1}$, it suffices to show that an alternating product in $\left\{v_{1 j}, v_{j 1}\right\}$ and $\mathcal{N}_{1}$ that has $m$ more copies of $v_{1 j}$ than of $v_{j 1}$ has trace zero. But we know that every element of $\mathcal{N}_{1}$ is the s.o.-limit of a bounded sequence in $\operatorname{span} \Lambda\left(\{a\},\left\{b_{1}, \ldots, b_{n}\right\}\right)$. So it suffices to show that an alternating product in $\left\{v_{1 j}, v_{j 1}\right\}$ and $\Lambda\left(\{a\},\left\{b_{1}, \ldots, b_{n}\right\}\right)$ that has $m$ more copies of $v_{1 j}$ than of $v_{j 1}$ has trace zero. But such a product can be reduced to give an alternating product in $\{a\}$ and $\left\{v_{1 j}, v_{j 1}, b_{1}, \ldots, b_{n}\right\}$, which must be nontrivial because there were more $v_{1 j}$ than $v_{j 1}$, so by freeness has trace zero. This proves the claim. 
Claim 3.2c. $\left\{h_{1} \mathcal{N}_{2} h_{1},\left(\left\{z_{j} v_{1 j} y_{j 1}\right\}\right)_{2 \leq j \leq n}\right\}$ is $*$-free in $h_{1} \mathcal{M} h_{1}$.

Indeed. Let $\mathcal{N}_{20}=\left(\left\{h_{1}\right\} \cup\left\{v_{1 j} h_{j} v_{j 1} \mid 2 \leq j \leq n\right\}\right)^{\prime \prime}$ (with identity element $\left.q_{1}\right)$. Let $Q=h_{1} \mathcal{N}_{1} h_{1}=L\left(\mathbf{F}_{n-1}\right)$ and $R=h_{1} \mathcal{N}_{20} h_{1}$. Then $Q$ and $R$ together generate $h_{1} \mathcal{N}_{2} h_{1}$ (and by $1.2,\{Q, R\}$ is free in $h_{1} \mathcal{N}_{2} h_{1}$. We will show that $\left\{Q, R,\left(\left\{z_{j} v_{1 j} y_{j 1}\right\}\right)_{2 \leq j \leq n}\right\}$ is free in $h_{1} \mathcal{M} h_{1}$. It suffices to show that a nontrivial traveling product in $\stackrel{o}{R}, \stackrel{o}{Q}$, $\left(\left\{\left(z_{j} v_{1 j} y_{j 1}\right)^{m} \mid m \in \mathbf{Z} \backslash\{0\}\right\}\right)_{2 \leq j \leq n}$ has trace zero. Regrouping gives a nontrivial alternating product, call it $\mathcal{P}$, in

$$
\Omega_{1}=\stackrel{o}{Q} \cup \bigcup_{2 \leq j \leq n}\left(Q y_{1 j} \cup y_{j 1} Q \cup y_{j 1} \stackrel{o}{Q} y_{1 j}\right) \cup \bigcup_{\substack{2 \leq i, j \leq n \\ i \neq j}}^{\bigcup} y_{i 1} Q y_{1 j}
$$

and

$$
\Omega_{2}=\stackrel{o}{R} \cup \bigcup_{2 \leq j \leq n}\left(v_{j 1} z_{j}^{*} R \cup R z_{j} v_{1 j} \cup v_{j 1} z_{j}^{*} R z_{j} v_{1 j}\right) \cup \bigcup_{\substack{2 \leq i, j \leq n \\ i \neq j}} v_{j 1} z_{j}^{*} R z_{i} v_{1 i}
$$

where an occurrence of $y_{1 j}$ (be it from $Q y_{1 j}, y_{j 1} \stackrel{o}{Q} y_{1 j}$ or $y_{i 1} Q y_{1 j}$ ) is always followed by an occurrence of $v_{j 1} z_{j}^{*}$ (be it from $v_{j 1} z_{j}^{*} R, v_{j 1} z_{j}^{*} \stackrel{o}{R} z_{j} v_{1 j}$ or $\left.v_{j 1} z_{j}^{*} R z_{i} v_{1 i}\right)$. We similarly have the list of conditions:

$\begin{array}{cccccc}\text { An occurrence of } & y_{1 j} & \text { is always followed by an occurrence of } v_{j 1} z_{j}^{*} \text {, } \\ " & y_{j 1} & & \text { preceded by } & " & z_{j} v_{1 j}, \\ " & z_{j} v_{1 j} & " & \text { followed by } & " & y_{j 1}, \\ " & v_{j 1} z_{j}^{*} & & \text { preceded by } & " & y_{1 j} .\end{array}$

Let $d, c_{j}, \tilde{c}_{j}$ be as in the proof of Claim 3.2b, and denote also $d=c_{1}$. Then $\left\{\left(c_{j}\right)_{1 \leq j \leq n}\right\}$ is free in $h_{1} \mathcal{M} h_{1}$ (as we saw) and $\operatorname{span} \Lambda\left(\left(\left\{c_{j}\right\}\right)_{1 \leq j \leq n}\right)$ is a dense ${ }^{*}$-subalgebra of $\mathcal{N}_{20}$. For $1 \leq k, l \leq n$, let $W_{k l}$ be the set of nontrivial traveling products in $\left(c_{i}\right)_{1 \leq i \leq n}$ which begin with a letter other than $c_{k}$ and end with a letter other than $c_{l}$. If $x \in \stackrel{o}{R}$, then $\mathrm{x}$ is the s.o.-limit of a bounded sequence in $h_{1} \operatorname{span}\left(W_{11}\right) h_{1}$. And since $z_{j} \in \mathcal{N}_{20}$, an element $x \in R z_{j}$ is the s.o.-limit of a bounded sequence in $h_{1} \operatorname{span}\left(\{1\} \cup W_{1 j}\right) v_{1 j} h_{j} v_{j 1}$, so every element of $R z_{j} v_{1 j}$ is the s.o.-limit of a bounded sequence in $h_{1} \operatorname{span}\left(\{1\} \cup W_{1 j}\right) v_{1 j} h_{j}$. In a similar way we obtain approximating sequences for the other elements of $\Omega_{2}$, and hence deduce that it suffices to show that we get something of trace zero when we substitute in our alternating product $\mathcal{P}$

$$
\begin{aligned}
& \text { for every occurrance of a letter from } \stackrel{o}{R} \text { an arbitrary element of } h_{1} W_{11} h_{1} \text {, } \\
& \begin{array}{llll} 
& R z_{j} v_{1 j} \quad " & & h_{1}\left(W_{1 j} \cup\{1\}\right) v_{1 j} h_{j}, \\
& v_{j 1} z_{j}^{*} R \quad " & & h_{j} v_{j 1}\left(W_{j 1} \cup\{1\}\right) h_{1}, \\
" & v_{j 1} z_{j}^{*} R z_{j} v_{1 j} \quad " & & h_{j} v_{j 1}\left(W_{j j}\right) v_{1 j} h_{j} \\
& v_{j 1} z_{j}^{*} R z_{i} v_{1 i} \quad " & & h_{j} v_{j 1}\left(W_{j i} \cup\{1\}\right) v_{1 i} h_{i}(i \neq j) .
\end{array}
\end{aligned}
$$

When making the above substitutions, we do not need to worry about writing the $h_{1}, h_{j}$ or $h_{i}$ (from (15)) because the neighboring element from $\Omega_{1}$ will absorb it, except possibly for an $h_{1}$ at the very beginning and one at the very end. If an $h_{1}$ occurs at the beginning or the end, write $h_{1}=d+n(1-\alpha) q_{1}$ and distribute, giving a sum of up to four products. Now write each element of $W_{k l}(1 \leq k, l \leq n)$ as a word in $\left(\left\{c_{i}\right\}\right)_{1 \leq i \leq n}$, and for each $c_{i}$ with $i \geq 2$ write $c_{i}=v_{1 i} \tilde{c}_{i} v_{i 1}$. But every element of $\Omega_{1}$ is the s.o.-limit of a bounded sequence in $\operatorname{span}\left(\Lambda\left(\{a\},\left\{b_{1}, \ldots, b_{n}\right\}\right) \backslash\left\{1, b_{1}, \ldots, b_{n}\right\}\right)$, as is $c_{1}$ and each $\tilde{c}_{i}(2 \leq i \leq n)$. So it suffices to show that replacing each letter from $\Omega_{1}$, each $c_{1}$ and each $\tilde{c}_{i}(2 \leq i \leq n)$ with an arbitrary element of $\Lambda\left(\{a\},\left\{b_{1}, \ldots, b_{n}\right\}\right) \backslash\left\{1, b_{1}, \ldots, b_{n}\right\}$, we get something having trace zero. But because of the rules (14) about neighbors, we see that by regrouping, we get a nontrivial alternating product in $\{a\}$ and $\left\{b_{1}, \ldots, b_{n}\right\} \cup\left\{v_{i j} \mid i \neq j\right\}$, which by freeness has trace zero. This proves Claim 3.2c.

From (13) and Claims $3.2 \mathrm{~b}$ and c we can find $\mathcal{M}$ in the two cases to finish the proof of the proposition. 
It may be of interest to note that the projection $p^{\prime}$ in the statement of the proposition above is

$$
p^{\prime}=\sum_{1 \leq j \leq n}\left(p \wedge q_{j}\right) \wedge \bigwedge_{\substack{1 \leq i \leq n \\ i \neq j}} v_{j i}\left(p \wedge q_{i}\right) v_{i j} \leq p .
$$

Now we have all that we need to determine the free product of any given pair of finite dimensional algebras. It is most natural to phrase the results in terms of free dimension.

Definition 3.3. The free dimension of a finite dimensional algebra

$$
A=\underset{\alpha_{1}}{M_{n_{1}}} \oplus \cdots \oplus \underset{\alpha_{k}}{M_{n_{k}}}(k \geq 1)
$$

is equal to

$$
\operatorname{fdim}(A)=\sum_{1 \leq i \leq k} \alpha_{i}^{2}\left(1-n_{i}^{-2}\right)+\sum_{\substack{1 \leq i, j \leq k \\ i \neq j}} \alpha_{i} \alpha_{j} .
$$

More generally, the free dimension of an algebra

$$
B=\underset{\alpha_{0}}{L\left(\mathbf{F}_{s}\right)} \underset{\alpha_{1}}{M_{n_{1}}} \oplus \cdots \oplus \underset{\alpha_{k}}{M_{n_{k}}}(k \geq 0, s \geq 1)
$$

is equal to

$$
\operatorname{fdim}(B)=\alpha_{0}^{2} s+\sum_{1 \leq i \leq k} \alpha_{i}^{2}\left(1-n_{i}^{-2}\right)+\sum_{\substack{0 \leq i, j \leq k \\ i \neq j}} \alpha_{i} \alpha_{j} .
$$

The arguments leading up tp Theorem 3.6 are once again essentially nothing more than induction resting on the shoulders of the results we have already proven. There are certainly many different ways to formally write down this procedure. The following sequence of lemmas outlines one such proof. Once again, we will omit the laborious and elementary algebraic verifications.

Lemma 3.4. Let

$$
\mathcal{A}=L \underset{\alpha_{0}}{L\left(\mathbf{F}_{r}\right)} \oplus \underset{\alpha_{1}}{\mathbf{C}} \oplus \cdots \oplus \underset{\alpha_{k}}{p_{k}}\left(k \geq 0, r \geq 1, \alpha_{0} \geq 0\right),
$$

where $0<\alpha_{1} \leq \alpha_{2} \leq \cdots \leq \alpha_{k}$, and let $m \geq 2$. Then

$$
\mathcal{A} * M_{m} \cong \begin{cases}L\left(\mathbf{F}_{s}\right) & \text { if } \alpha_{k} \leq 1-m^{-2} \\ & \text { if } \alpha_{k} \geq 1-m^{-2},\end{cases}
$$

where $s$ is such that the right hand side of (16) has free dimension equal to $\operatorname{fdim}(\mathcal{A})+1-m^{-2}$, and where $p^{\prime} \leq p_{k}$.

Proof. Let $\mathcal{M}$ be the left hand side of (16). The case $k=0$ follows from 3.1 and 2.4 , as does the case $k \geq 1$, $\alpha_{k} \leq 1-m^{-1}$. The case $m^{-2} \leq \alpha_{k}$ follows from $1.2,2.4$ and 3.2 by writing

$$
\begin{aligned}
& \mathcal{M}=\left(\mathcal{B} \oplus \underset{\alpha_{k}}{\mathbf{C}}\right) * M_{m} \\
& \cup \\
& \mathcal{N}=\left(\mathbf{C} \oplus \boldsymbol{\alpha}_{\alpha_{k}}^{\mathbf{C}}\right) * M_{m}
\end{aligned}
$$

Similarly one can prove 
Proposition 3.5. Let

$$
\mathcal{A}=\underset{\alpha_{0}}{L\left(\mathbf{F}_{r}\right)} \underset{\alpha_{1}}{\stackrel{p_{1}}{M}} \underset{\alpha_{1}}{\stackrel{p_{k}}{M}} \oplus \underset{\alpha_{k}}{M_{n_{k}}}\left(k \geq 0, r \geq 1, \alpha_{0} \geq 0\right)
$$

and let $m \geq 2$. If $n_{j} \geq 2$ for all $1 \leq j \leq k$, then $\mathcal{A} * M_{m}=L\left(\mathbf{F}\left(\operatorname{fdim}(\mathcal{A})+1-m^{-2}\right)\right)$. If some $n_{j}=1$, assume that $n_{k}=1$ and $\alpha_{k}=\max \left\{\alpha_{j} \mid 1 \leq j \leq k, n_{j}=1\right\}$. Then

$$
\mathcal{A} * M_{m}= \begin{cases}L\left(\mathbf{F}\left(\operatorname{fdim}(\mathcal{A})+1-m^{-2}\right)\right) & \text { if } \alpha_{k} \leq 1-m^{-2} \\ L\left(\mathbf{F}_{s}\right) \oplus \underset{p^{\prime}}{M_{m} \alpha_{k}-m^{2}+1} & \text { if } \alpha_{k} \geq 1-m^{-2}\end{cases}
$$

where $p^{\prime} \leq p_{k}$ and $s$ is such that the free dimension of the right hand side of $(17)$ is equal to $\operatorname{fdim}(\mathcal{A})+1-m^{-2}$.

Theorem 3.6. Let

$$
\begin{aligned}
& \stackrel{p_{1}}{M}{ }_{n_{1}} \oplus \cdots \oplus \stackrel{p_{k}}{M_{n_{k}}} \\
& B=\stackrel{q_{1}}{M_{m_{1}}} \oplus \cdots \oplus \underset{\beta_{l}}{M_{m_{l}}^{q_{l}}}
\end{aligned}
$$

be finite dimensional algebras, each of dimension $\geq 2$ and such that the sum of their dimensions is $\geq 5$. Then

$$
A * B=L\left(\mathbf{F}_{s}\right) \oplus \bigoplus_{\substack{1 \leq i \leq k \\ 1 \leq j \leq l}}{\stackrel{f_{i j}}{\gamma_{i j}}}_{N(i, j)}
$$

where $N(i, j)=\max \left(n_{i}, m_{j}\right), \gamma_{i j}=N(i, j)^{2} \max \left(\frac{\alpha_{i}}{n_{i}^{2}}+\frac{\beta_{j}}{m_{j}^{2}}-1,0\right)$ and where $f_{i j} \leq p_{i} \wedge q_{j}$. Note that $\gamma_{i j}>0$ implies either $n_{i}=1$ or $m_{j}=1$.

Proof. By induction on $K=\operatorname{card}\left(\left\{n_{i} \geq 2\right\} \cup\left\{m_{j} \geq 2\right\}\right)$. Let $\mathcal{M}$ be the left hand side of (18). The case $K=0$ is just 2.3. For the inductive step, let $K \geq 1$ and suppose $n_{k} \geq 2$. Write $A=A_{0} \oplus M_{n_{k}}$,

$$
\begin{aligned}
& \mathcal{M}=\left(\begin{array}{c}
A_{0} \oplus M_{n_{k}} \\
\alpha_{k}
\end{array}\right) B \\
& \cup \\
& \mathcal{N}=\left(A_{0} \oplus \underset{\alpha_{k}}{\mathbf{C}}\right) * B
\end{aligned}
$$

and use 1.2, the inductive hypothesis and 3.5.

\section{$\S 4$. Hyperfinite algebras.}

In this section we will extend the results of the last section to hyperfinite von Neumann algebras, i.e. inductive limits of finite dimensional algebras (with trace-preserving morphisms).

Definition 4.1. Let $\mathcal{A}=L\left(\mathbf{F}_{r}\right), \mathcal{B}=L\left(\mathbf{F}_{r^{\prime}}\right)$ with $r<r^{\prime}$. Then $\psi: \mathcal{A} \rightarrow \mathcal{B}$ is a standard embedding if the following is satisfied: for some $\mathrm{W}^{*}$-noncommutative probability space $(\mathcal{M}, \phi)$ with $\phi$ a trace and with $\mathcal{M}$ containing a copy $R$ of the hyperfinite $\mathrm{II}_{1}$-factor and a semicircular family $\omega=\left\{X^{t} \mid t \in T\right\}$ such that $\{R, \omega\}$ is free, there exist subsets $S \subset S^{\prime} \subseteq T$, projections $p_{s} \in R\left(s \in S^{\prime}\right)$ and isomorphisms $\alpha: \mathcal{A} \rightarrow\left(R \cup\left\{p_{s} X^{s} p_{s} \mid s \in S\right\}\right)^{\prime \prime}$ and $\beta: \mathcal{B} \rightarrow\left(R \cup\left\{p_{s} X^{s} p_{s} \mid s \in S^{\prime}\right\}\right)^{\prime \prime}$ such that $\psi=\beta^{-1} \circ i \circ \alpha$, where $i$ is the inclusion.

If $\psi: \mathcal{A} \rightarrow \mathcal{B}$ is an injective morphism of von Neumann algebras, $p \in \mathcal{A}$ a projection, then $\left.\psi\right|_{p \mathcal{A} p}: p \mathcal{A} p \rightarrow$ $\psi(p) \mathcal{B} \psi(p)$ is also an injective morphism of von Neumann algebras. We have 
Proposition 4.2. Let $\mathcal{A}=L\left(\mathbf{F}_{r}\right), \mathcal{B}=L\left(\mathbf{F}_{r^{\prime}}\right)$ with $r<r^{\prime}$; suppose $\psi: \mathcal{A} \rightarrow \mathcal{B}, p \in \mathcal{A}$ a projection. Then $\psi$ is a standard embedding if and only if $\left.\psi\right|_{p \mathcal{A} p}$ is a standard embedding.

Proof. Since tensoring with $M_{n}$ preserves "standardness," it is enough to prove one direction. Suppose $\psi$ is a standard embedding and let $\alpha, \beta$ be isomorphisms as in Definition 4.1. By the proof of 2.2 of [4], we may assume that $p_{s} \leq p$ for all $s \in S^{\prime}$. Then

$$
\begin{aligned}
\left.\alpha\right|_{p \mathcal{A} p} & : p \mathcal{A} p \stackrel{\sim}{\rightarrow}\left(p R p \cup\left\{p_{s} X^{s} p_{s} \mid s \in S\right\}\right)^{\prime \prime} \\
\left.\beta\right|_{\psi(p) \mathcal{B} \psi(p)} & : \psi(p) \mathcal{B} \psi(p) \stackrel{\sim}{\rightarrow}\left(p R p \cup\left\{p_{s} X^{s} p_{s} \mid s \in S^{\prime}\right\}\right)^{\prime \prime}
\end{aligned}
$$

and $\left.\psi\right|_{p \mathcal{A} p}=\left(\left.\beta\right|_{\psi(p) \mathcal{B} \psi(p)}\right)^{-1} i\left(\left.\alpha\right|_{p \mathcal{A} p}\right)$. Theorem 1.3 of [4] tells us that the generators on the right hand side of (19) are of the correct sort, so $\left.\psi\right|_{p \mathcal{A} p}$ is a standard embedding.

\section{Proposition 4.3.}

(i) The composition of standard embeddings is a standard embedding.

(ii) Let $\mathcal{A}_{n}=L\left(\mathbf{F}_{r_{n}}\right)$ for $n \geq 1$ with $r_{n}<r_{n+1}, \psi_{n}: \mathcal{A}_{n} \rightarrow \mathcal{A}_{n+1}$ standard embeddings. Then $\underset{n}{\lim }\left(\mathcal{A}_{n}, \psi_{n}\right)=$ $L\left(\mathbf{F}_{r}\right)$ where $r=\lim _{n \rightarrow \infty} r_{n}$.

Proof. To prove (i), let $\mathcal{A}=L\left(\mathbf{F}_{r}\right), \mathcal{B}=L\left(\mathbf{F}_{s}\right)$ and $\mathcal{C}=L\left(\mathbf{F}_{t}\right)$ for $r<s<t$. Let $\psi: \mathcal{A} \rightarrow \mathcal{B}$ and $\widetilde{\psi}: \mathcal{B} \rightarrow \mathcal{C}$ be standard embeddings. Then taking $(\mathcal{M}, \phi),\left\{X^{t} \mid t \in T\right\}$ as in Definition 4.1, there exist isomorphisms

$$
\begin{aligned}
& \alpha: \mathcal{A} \stackrel{\sim}{\rightarrow}\left(R \cup\left\{p_{s} X^{s} p_{s} \mid s \in S\right\}\right)^{\prime \prime} \\
& \beta: \mathcal{B} \stackrel{\sim}{\rightarrow}\left(R \cup\left\{p_{s} X^{s} p_{s} \mid s \in S^{\prime}\right\}\right)^{\prime \prime} \\
& \tilde{\beta}: \mathcal{B} \stackrel{\sim}{\rightarrow}\left(R \cup\left\{p_{s} X^{s} p_{s} \mid s \in \widetilde{S}\right\}\right)^{\prime \prime} \\
& \tilde{\gamma}: \mathcal{C} \stackrel{\sim}{\rightarrow}\left(R \cup\left\{p_{s} X^{s} p_{s} \mid s \in \widetilde{S}^{\prime}\right\}\right)^{\prime \prime},
\end{aligned}
$$

where $S \subset S^{\prime} \subset T, \widetilde{S} \subset \widetilde{S}^{\prime} \subset T, S^{\prime}$ and $\widetilde{S}^{\prime}$ are disjoint, $p_{s}$ are projections in $R$ and $\psi=\beta^{-1} \circ i \circ \alpha, \widetilde{\psi}=\tilde{\gamma}^{-1} \circ i \circ \tilde{\beta}$. Using the isomorphism $\beta \circ \tilde{\beta}^{-1}$ we get an isomorphism

$$
\bar{\gamma}: \mathcal{C} \stackrel{\sim}{\rightarrow}\left(R \cup\left\{p_{s} X^{s} p_{s} \mid s \in S^{\prime}\right\} \cup\left\{\tilde{p}_{s} X^{s} \tilde{p}_{s} \mid s \in \widetilde{S}^{\prime} \backslash \widetilde{S}\right\}\right)^{\prime \prime}
$$

where each $\tilde{p}_{s} \in\left(R \cup\left\{p_{s} X^{s} p_{s} \mid s \in S^{\prime}\right\}\right)^{\prime \prime}$ and such that $\widetilde{\psi} \circ \psi=\bar{\gamma}^{-1} \circ i \circ \alpha$. For $s \in \widetilde{S}^{\prime} \backslash \widetilde{S}$ let $U_{s} \in\left(R \cup\left\{p_{s} X^{s} p_{s} \mid\right.\right.$ $\left.\left.s \in S^{\prime}\right\}\right)^{\prime \prime}$ be a unitary such that $U_{s} \tilde{p}_{s} U_{s}^{*} \in R$. We see that $\left\{R,\left(\left\{X^{s}\right\}\right)_{s \in S^{\prime}},\left(\left\{U_{s} X^{s} U_{s}^{*}\right\}\right)_{s \in \tilde{S}^{\prime} \backslash \tilde{S}}\right\}$ is free and we may rewrite

$$
\bar{\gamma}: \mathcal{C} \stackrel{\sim}{\rightarrow}\left(R \cup\left\{p_{s} X^{s} p_{s} \mid s \in S^{\prime}\right\} \cup\left\{\left(U_{s} \tilde{p}_{s} U_{s}^{*}\right)\left(U_{s} X^{s} U_{s}^{*}\right)\left(U_{s} \tilde{p}_{s} U_{S}^{*}\right) \mid s \in \widetilde{S}^{\prime} \backslash \widetilde{S}\right\}\right)^{\prime \prime},
$$

thus proving (i).

In order to prove (ii), we may apply the above argument recursively to obtain $S_{1} \subset S_{2} \subset S_{3} \subset \cdots$ and isomorphisms

$$
\alpha_{n}: \mathcal{A}_{n} \stackrel{\sim}{\rightarrow}\left(R \cup\left\{p_{s} X^{s} p_{s} \mid s \in S_{n}\right\}\right)^{\prime \prime}
$$

such that $\psi_{n}=\alpha_{n+1}^{-1} \circ i \circ \alpha_{n}$. Then

$$
\underset{n}{\varliminf_{n}}\left(\mathcal{A}_{n}, \psi_{n}\right) \cong\left(R \cup\left\{p_{s} X^{s} p_{s} \mid s \in S_{1} \cup S_{2} \cup \cdots\right\}\right)^{\prime \prime}=L\left(\mathbf{F}_{r}\right)
$$


Proposition 4.4. The following are standard embeddings:

(i) the inclusion $i_{1}: L\left(\mathbf{F}_{r}\right) \hookrightarrow L\left(\mathbf{F}_{r}\right) * L\left(\mathbf{F}_{r^{\prime}}\right)$ for $1<r, r^{\prime} \leq \infty$;

(ii) the inclusion $i_{1}: L\left(\mathbf{F}_{r}\right) \hookrightarrow L\left(\mathbf{F}_{r}\right) * B$ for $1<r \leq \infty, B$ a finite dimensional algebra not equal to $\mathbf{C}$;

Proof. (i) In a noncommutative probability space $(\mathcal{M}, \phi)$ with $\phi$ a trace, let $R$ and $\widetilde{R}$ be copies of the hyperfinite $\mathrm{II}_{1}$-factor and $\omega=\left\{X^{t} \mid t \in T\right\}$ a semicircular family such that $\{R, \widetilde{R}, \omega\}$ is free. Let $S, \widetilde{S}$ be disjoint subsets of $T$ and $p_{s} \in R(s \in S), \tilde{p}_{s} \in \widetilde{R}(s \in \widetilde{S})$ be projections. Then it suffices to show that

$$
\left(R \cup\left\{p_{s} X^{s} p_{s} \mid s \in S\right\}\right)^{\prime \prime} \stackrel{i}{\hookrightarrow}\left(R \cup\left\{p_{s} X^{s} p_{s} \mid s \in S\right\} \cup \widetilde{R} \cup\left\{\tilde{p}_{s} X^{s} \tilde{p}_{s} \mid s \in \widetilde{S}\right\}\right)^{\prime \prime}
$$

is a standard embedding. By 3.6 of [4], there is a semicircular element $Y \in(R \cup \widetilde{R})^{\prime \prime}$ such that $\{R,\{Y\}, \omega\}$ is free and $(R \cup\{Y\})^{\prime \prime}=(R \cup \widetilde{R})^{\prime \prime}$. Then for each $s \in \widetilde{S}$ there is a unitary $U_{s} \in(R \cup\{Y\})^{\prime \prime}$ such that $U_{s} \tilde{p}_{s} U_{s}^{*}=q_{s} \in R$. It is easily seen that $\left\{R,\{Y\},\left(\left\{X^{s}\right\}\right)_{s \in S},\left(\left\{U_{s} X^{s} U_{s}^{*}\right\}\right)_{s \in \tilde{S}}\right\}$ is free. The right hand side of (20) equals $\left(R \cup\left\{p_{s} X^{s} p_{s} \mid\right.\right.$ $\left.s \in S\} \cup\{Y\} \cup\left\{q_{s}\left(U_{s} X^{s} U_{s}^{*}\right) q_{s} \mid s \in \widetilde{S}\right\}\right)^{\prime \prime}$, and the inclusion of (20) is then clearly a standard embedding.

(ii) Let $n \geq 2$ be so large that $M_{n} * B$ is a factor and let $\left\{e_{i j} \mid 1 \leq i, j \leq n\right\}$ be a system of matrix units in $\mathcal{A}=L\left(\mathbf{F}_{r}\right)$. Then $\mathcal{A}=\left(e_{11} \mathcal{A} e_{11}\right) \otimes M_{n}$. So

$$
\left.i_{1}\right|_{e_{11} \mathcal{A} e_{11}}: e_{11} \mathcal{A} e_{11} \hookrightarrow e_{11}(\mathcal{A} * B) e_{11}
$$

is by 1.2 conjugate to the inclusion

$$
e_{11} \mathcal{A} e_{11} \hookrightarrow\left(e_{11} \mathcal{A} e_{11}\right) *\left(e_{11}\left(M_{n} * B\right) e_{11}\right) .
$$

But by the compression formula (2) we have $e_{11} \mathcal{A} e_{11}=L\left(\mathbf{F}\left(1+n^{2}(r-1)\right)\right)$, and by 3.6 together with the compression formula we have $e_{11}\left(M_{n} * B\right) e_{11}=L\left(\mathbf{F}\left(n^{2} \operatorname{fdim}(B)\right)\right)$. Then by (i), (21) is a standard embedding, so by $4.2, \mathcal{A} \hookrightarrow \mathcal{A} * B$ is a standard embedding.

Let $\mathcal{A}$ be a hyperfinite von Neumann algebra (with implicitly specified trace). Then decomposing $\mathcal{A}$ over its center, we may write

$$
\mathcal{A}=\underset{\alpha}{\widetilde{\mathcal{A}}} \oplus \bigoplus_{i \in I} \stackrel{p_{i}}{M_{n_{i}}}
$$

where $\alpha \geq 0, I$ is a finite or countably infinite (or empty) index set, $n_{i} \in \mathbf{N} \backslash\{0\}$ and $\alpha_{i}>0$, and where $\widetilde{\mathcal{A}}$ is a diffuse von Neumann algebra, which can thus be further written as

$$
\left.\left.\widetilde{\mathcal{A}}=\underset{\gamma_{1}}{L(\mathbf{Z})} \oplus \underset{\gamma_{2}}{(L(\mathbf{Z}) \otimes} M_{2}\right) \oplus\left(\underset{\gamma_{3}}{(L(\mathbf{Z}) \otimes} M_{3}\right) \oplus \cdots \oplus \underset{\gamma_{\infty}}{(L(\mathbf{Z})} \otimes R\right) \oplus \underset{j \in J}{\bigoplus} \underset{\delta_{j}}{R}
$$

where $R$ is the hyperfinite $\mathrm{II}_{1}$-factor, $\gamma_{k} \geq 0, J$ is a finite or countably infinite (or empty) index set and $\delta_{j}>0$.

Definition 4.5. The free dimension of a hyperfinite von Neumann algebra $\mathcal{A}$ written as in (22) is equal to

$$
\operatorname{fdim}(\mathcal{A})=\alpha^{2}+\sum_{i \in I} \alpha_{i}^{2}\left(1-n_{i}^{-2}\right)+2 \alpha(1-\alpha)+\sum_{\substack{i, j \in I \\ i \neq j}} \alpha_{i} \alpha_{j}
$$

Theorem 4.6. Let $\mathcal{A}$ and $\mathcal{B}$ be hyperfinite (or finite dimensional) von Neumann algebras such that $\operatorname{dim}(\mathcal{A}), \operatorname{dim}(\mathcal{B}) \geq$ 2 and $\operatorname{dim}(\mathcal{A})+\operatorname{dim}(\mathcal{B}) \geq 5$. Let $\mathcal{A}$ be written as in (22) and write

$$
\mathcal{B}=\underset{\beta}{\widetilde{\mathcal{B}}} \oplus \bigoplus_{j \in J} \stackrel{q_{j}}{M_{m_{j}}}
$$


where $\beta \geq 0, J$ is a finite or countably infinite (or empty) index set, $m_{j} \in \mathbf{N} \backslash\{0\}$ and $\beta_{j}>0$, and where $\widetilde{\mathcal{B}}$ is a diffuse von Neumann algebra. Then

$$
\mathcal{A} * \mathcal{B} \cong L\left(\mathbf{F}_{\gamma}\right) \oplus \bigoplus_{\substack{i \in I \\ j \in J}} M_{\gamma_{i j}}^{f_{i j}},
$$

where $N(i, j)=\max \left(n_{i}, m_{j}\right), \gamma_{i j}=N(i, j)^{2} \max \left(\frac{\alpha_{i}}{n_{i}^{2}}+\frac{\beta_{j}}{m_{j}^{2}}-1,0\right)$ and $f_{i j} \leq p_{i} \wedge q_{j}$, and where $s$ is such that the free dimension of the right hand side of (23) equals $\operatorname{fdim}(\mathcal{A})+\operatorname{fdim}(\mathcal{B})$. Note that $\gamma_{i j}>0$ implies either $n_{i}=1$ or $m_{i}=1$, and that there are only finitely many pairs $(i, j) \in I \times J$ for which $\gamma_{i j}>0$.

Proof. Let $\left(A_{k}\right)_{k \geq 1}$ be an increasing sequence of finite dimensional subalgebras of $\mathcal{A}$ such that $\cup A_{k}$ is dense in $\mathcal{A}$ and such that each inclusion $A_{k} \hookrightarrow A_{k+1}$ (which we denote $\phi_{k}$ ) is of the form $A_{k}=M_{n} \oplus A \hookrightarrow\left(M_{n} \otimes C\right) \oplus A=A_{k+1}$, where $A$ and $C$ are finite dimensional algebras (depending on $k$ ). Then $\operatorname{fdim}\left(A_{k}\right)$ is a non-decreasing sequence whose limit is $\operatorname{fdim}(\mathcal{A})$. (If $\mathcal{A}$ is finite dimensional then eventually the $A_{k}$ are all the same.) Let $\left(B_{k}\right)_{k \geq 1}$ be a similar sequence for $\mathcal{B}$, and denote the inclusion $B_{k} \hookrightarrow B_{k+1}$ by $\psi_{k}$. Theorem 3.6 allows us to find each $A_{k} * B_{k}$, and for large enough $k$, we have that

$$
A_{k} * B_{k}=L\left(\mathbf{F}_{s_{k}}\right) \oplus \bigoplus_{\substack{i \in I \\ j \in J}} M_{\gamma_{i j}}^{f_{i j}},
$$

with $N(i, j)$ and $\gamma_{i j}$ as in (23). Moreover, letting $f=1-\sum_{i \in I, j \in J} f_{i j}$, we see that $\left.\phi_{k} * \psi_{k}\right|_{(1-f)\left(A_{k} * B_{k}\right)(1-f)}$ is the identity map on the finite dimensional part $\bigoplus M_{N(i j)}$. Using 1.4, 4.2, 4.3(i) and 4.4(ii), we can show that $\left.\phi_{k} * \psi_{k}\right|_{f\left(A_{k} * B_{k}\right) f}: L\left(\mathbf{F}_{s_{k}}\right) \hookrightarrow L\left(\mathbf{F}_{s_{k+1}}\right)$ is a standard embedding. Taking inductive limits and applying 4.3(ii) proves the theorem.

Remark 4.7. The precise condition for $\mathcal{A} * \mathcal{B}$ to be a factor may be described as follows. Let $\mathcal{A}$ be as written in (22) and define the lumpiness of $\mathcal{A}$ to be $l_{\mathcal{A}}=\max \left\{\frac{\alpha_{i}}{n_{i}^{2}} \mid i \in I\right\}$. Let $l_{\mathcal{B}}$ be the lumpiness of $\mathcal{B}$. Then $\mathcal{A} * \mathcal{B}$ is a factor if and only if $l_{\mathcal{A}}+l_{\mathcal{B}} \leq 1$, (provided that neither $\mathcal{A}$ nor $\mathcal{B}$ is one-dimensional and one of them is $\geq 3$-dimensional).

\section{$\S 5$. Group algebras.}

In the fundamental paper [2], A. Connes showed that $L(G)$ is hyperfinite for $G$ a discrete amenable group. It is now easy to find the free dimension of such $L(G)$, and to that end we want to have the following proposition which has been known for some time. ${ }^{1}$

Proposition 5.1. Let $G$ be an infinite discrete group. Then $L(G)$ is diffuse.

Proof. Suppose for contradiction that $p \in L(G)$ is a central projection having trace $\alpha>0$ and that $p L(G) \cong M_{n}$. Then $p\left(l^{2}(G)\right) \cong L^{2}\left(p L(G), \alpha^{-1} \tau\right) \cong L^{2}\left(M_{n}, \tau_{2}\right)$, (where $\tau$ is the canonical trace on $L(G), \tau_{2}$ the normalized trace on $\left.M_{n}\right)$, so $p\left(l^{2}(G)\right)$ has dimension $n^{2}$. Let $U_{g}$ for $g \in G$ denote the left translation operator on $l^{2}(G)$ and let $v_{1}, \ldots, v_{n^{2}}$ be an orthonormal basis for $p\left(l^{2}(G)\right)$. Then since $v_{i}, v_{j} \in l^{2}(G)$ we have $\left\langle U_{g} v_{i}, v_{j}\right\rangle \rightarrow 0$ as $g \rightarrow \infty$. But this contradicts the fact that $\left.U_{g}\right|_{p\left(l^{2}(G)\right)}$ is unitary for all $g \in G$.

Proposition 5.2. Let $G$ be an amenable discrete group. Then $L(G)$ has free dimension equal to $1-|G|^{-1}$ (where $\left.\infty^{-1}=0\right)$.

Proof. For $|G|=\infty$, we have by 5.1 that $L(G)$ is a diffuse hyperfinite von Neumann algebra, so has free dimension equal to 1 . For $|G|<\infty$, it is well-known that

$$
L(G)=\bigoplus_{\alpha \in \widehat{G}} M_{n_{\alpha}}
$$

\footnotetext{
${ }^{1}$ We would like to thank Sorin Popa: when asked if this result was true, he communicated to us that he had proved it in 1981, and he made helpful comments which enabled us to find this proof.
} 
where $\widehat{G}$ is the collection of equivalence classes of irreducible representations of $G, n_{\alpha}$ is the dimension of the representation and the tracial weight $\gamma_{\alpha}$ equals $n_{\alpha}^{2} /|G|$. One then uses 3.3.

Corollary 5.3. Let $G$ and $H$ be discrete amenable groups. Then $L(G * H)$ depends only on the orders of $G$ and $H$. More precisely,

$$
L(G * H)=L\left(\mathbf{F}\left(2-|G|^{-1}-|H|^{-1}\right)\right) \quad\left(\text { where } \infty^{-1}=0\right)
$$

provided $|G|,|H| \geq 2$ and $|G|+|H| \geq 5$.

Corollary 5.4. ${ }^{2}$ Let $G_{n}(n=1,2, \ldots)$ be nontrivial discrete amenable (or finite) groups. Then

$$
L\left(\stackrel{\infty}{*} G_{n=1}\right)=L\left(\mathbf{F}_{\infty}\right)
$$

Proof. $\mathcal{A}=L\left(\stackrel{\infty}{*} G_{n=1}\right)={ }_{k=1}^{*} L\left(G_{2 k-1} * G_{2 k}\right)$. Since $\mathbf{Z}_{2} * \mathbf{Z}_{2}$ is amenable, we may assume without loss of generality that for no $k$ are both $G_{2 k-1}$ and $G_{k}$ of order two. Then $G_{2 k-1} * G_{2 k}=L\left(\mathbf{F}\left(2-\left|G_{2 k-1}\right|^{-1}-\left|G_{2 k}\right|^{-1}\right)\right.$ and $L\left(\mathbf{F}\left(2-\left|G_{2 k-1}\right|^{-1}-\left|G_{2 k}\right|^{-1}\right)=\left(R_{k} \cup\left\{p_{k} X_{k} p_{x}\right\}\right)^{\prime \prime}\right.$ where $R_{k}$ is a copy of the hyperfinite $\mathrm{II}_{1}$-factor, $p_{k} \in R_{k}$ is a projection, $X_{k}$ is a semicircular element and $\left\{R_{k},\left\{X_{k}\right\}\right\}$ is free. Thus $\mathcal{A} \cong\left(\bigcup_{k=1}^{\infty} R_{k} \cup\left\{p_{k} X_{k} p_{k} \mid k \geq 1\right\}\right)^{\prime \prime}$ where

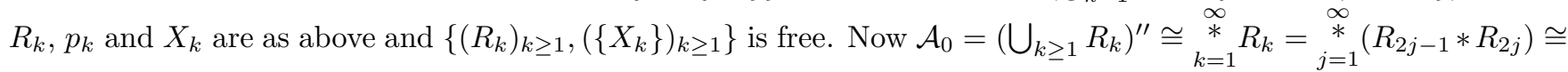
$\stackrel{\infty}{*} L\left(\mathbf{F}_{2}\right)=L\left(\mathbf{F}_{\infty}\right)=\left(R \cup\left\{Y_{l} \mid l \geq 1\right\}\right)^{\prime \prime}$ where $R$ is a copy of the hyperfinite $\mathrm{II}_{1}$-factor, $Y_{l}$ are semicircular elements and $\left\{R,\left(\left\{Y_{l}\right\}\right)_{l \geq 1}\right\}$ is free. For each $k$ there is a unitary $U_{k} \in \mathcal{A}_{0}$ such that $U_{k} p_{k} u_{k}^{*} \in R$. Then $\mathcal{A} \cong\left(R \cup\left\{Y_{l} \mid l \geq\right.\right.$ $\left.1\} \cup\left\{\left(U_{k} p_{k} U_{k}^{*}\right)\left(U_{k} X_{k} U_{k}^{*}\right)\left(U_{k} p_{k} U_{k}^{*}\right) \mid k \geq 1\right\}\right)^{\prime \prime}$ and it is easily seen that $\left\{R,\left(\left\{Y_{l}\right\}\right)_{l \geq 1},\left(\left\{U_{k} X_{k} U_{k}^{*}\right\}\right)_{k \geq 1}\right\}$ is free, so (by Defenition 2.1 of [4]) $\mathcal{A}=L\left(\mathbf{F}_{\infty}\right)$.

\section{Acknowledgements.}

I would like to thank Dan Voiculescu, my advisor, for helpful comments, also Dietmar Bisch for helpful conversations, Florin Boca for conversations about free products of finite dimensional abelian algebras and his (correct) conjecture about when they are factors and Sorin Popa for helpful comments about group algebras.

\section{REFERENCES}

1'. F. Boca, On the method of constructing irreducible finite index subfactors of Popa, preprint U.C.L.A., December 1991.

1. W.M. Ching, Free products of von Neumann algebras, Trans. Amer. Math. Soc. 178 (1973), $147-163$.

2. A. Connes, Classification of injective factors, Ann. of Math. 104 (1976), 73-115.

3. K. Dykema, On certain free product factors via an extended matrix model, J. Funct. Anal. (to appear).

4. Interpolated free group factors, Pacific J. Math. (to appear).

5. F.J. Murray and J. von Neumann, Rings of operators. IV, Ann. of Math. 44 (1943), $716-808$.

6. S. Popa, Markov traces on universal Jones algebras and subfactors of finite index, preprint I.H.E.S., June 1991.

7. F. Rădulescu, The fundamental group of the von Neumann algebra of a free group with infinitely many generators is $\mathbf{R}_{+} \backslash\{0\}$, J. Amer. Math. Soc. (to appear)

8. _ Stable isomorphism of the weak closure of free group convolution algebras, preprint, I.H.E.S., December, 1991.

9. _ Random matrices, amalgamated free products and subfactors of the von Neumann algebra of a free group, preprint, I.H.E.S., December, 1991.

10. D. Voiculescu, Symmetries of some reduced free product $C^{*}$-algebras, Operator Algebras and Their Connections with Topology and Ergodic Theory, Lecture Notes in Mathematics, Volume 1132, Springer-Verlag, 1985, pp. 556-588.

11. _ Multiplication of certain non-commuting random variables, J. Operator Theory 18 (1987), $223-235$.

12. Noncommutative random variables and spectral problems in free product $C^{*}-$ algebras, Rocky Mt. J. Math. 20 (1990), 263-283.

13. _ Circular and semicircular systems and free product factors, Operator Algebras, Unitary Representations, Enveloping Algebras, and Invariant Theory, Progress in Mathematics, Volume 92, Birkhäuser, Boston, 1990.

14. L_ Limit laws for Random matrices and free products, Invent. Math. 104 (1991), $201-220$.

15. Free non-commutative random variables, random matrices and the $I I_{1}$-factors of free groups, Quantum Probability and Related Topics VI, L. Accardi, ed., World Scientific, Singapore, 1991, pp. 473-487.

\footnotetext{
${ }^{2}$ Thanks to Florin Boca for pointing this out after reading a preliminary version of this paper.
} 